\title{
Temporal delays in trauma craniotomies
}

\author{
Judith Marcoux, MD, MSc, FRCSC, ${ }^{1,2}$ David Bracco, MD, ${ }^{3}$ and Rajeet S. Saluja, MD, FRCSC 1,2 \\ 'Department of Neurology and Neurosurgery, McGill University; and Departments of ${ }^{2}$ Neurosurgery and ${ }^{3}$ Anesthesia, McGill \\ University Health Centre, Montreal, Quebec, Canada
}

\begin{abstract}
OBJECTIVE The Brain Trauma Foundation recommendation regarding the timing of surgical evacuation of epidural hematomas and subdural hematomas is to perform the procedure as soon as possible. Indeed, faster evacuation is associated with better outcome. However, to the authors' knowledge, no study has looked at where delays in intrahospital care occurred for patients suffering from traumatic intracranial mass lesions. The goals of this study were as follows: 1) to characterize the performance of a Level 1 trauma center in terms of delays for emergency trauma craniotomies, 2) to review step by step where delays occurred in patient care, and 3) to propose ways to improve performance.
\end{abstract}

METHODS A retrospective review was conducted covering a 5-year period of all emergency trauma craniotomies. Demographic data, injury severity, neurological status, and functional outcome data were collected. The time elapsed between emergency department (ED) arrival and CT imaging, between CT imaging and arrival at the operating room $(\mathrm{OR})$, between ED arrival and OR arrival, between OR arrival and skin incision, and between ED arrival and skin incision were calculated. Patients were also subcategorized as either having immediate life-threatening emergencies (E0) or lifethreatening emergencies (E1). The operative technique was also reviewed (standard craniotomy opening vs immediate bur hole decompression followed by craniotomy).

RESULTS The study included 166 patients. Of these, 58 (35\%) were classified into the E0 group and 108 (64.2\%) into the E1 group. The median ED-to-CT delay was 54 minutes with no significant difference between the E0 and the E1 groups. The median CT-to-OR time delay was 57 minutes. The median delay for the E0 group was 39 minutes and that for the E1 group was 70 minutes $(p=0.002)$. The median delay from ED to OR arrival for patients with a CT scanning done at an outside hospital was 75 minutes. The median delay from ED to OR arrival was 85 minutes for the E0 group and 127 minutes for the E1 group ( $p<0.0001)$. The median delay from OR arrival to skin incision was 35 minutes (E0: median 27 minutes; E1: median 39 minutes; $p<0.0001$ ). The median total time elapsed between ED arrival and skin incision was 150 minutes (E0: median 131 minutes; E1: median 180 minutes). Overall, only $17 \%$ of patients underwent immediate bur hole decompression, but the proportion climbed to $41 \%$ in the E0 group. A lower Glasgow Coma Scale score was associated with a shorter delay $(p=0.0004)$.

CONCLUSIONS A long delay until surgery still exists for patients requiring urgent mass lesion evacuation. Many factors contribute to this delay, including performing imaging and transfer to and preparation in the OR. Strategies can be implemented to reduce delays and improve the delivery of care.

http://thejns.org/doi/abs/10.3171/2015.6.JNS15175

KEY WORDS traumatic brain injury; trauma craniotomy; acute epidural hematoma; acute subdural hematoma; quality of care; delay in treatment; surgery

667 IME is brain" for stroke victims awaiting revascularization with thrombolysis. ${ }^{5}$ In traumatic brain injury (TBI), rapidly growing mass lesions also lead to brain ischemia and damage. Rapid decompression of an expanding mass lesion has been thought of as a standard of care in neurosurgery. Surgical evacuation of an acute traumatic epidural hematoma (EDH) has been said to be "one of the most cost-effective of all surgical procedures in terms of quality of life and years preserved." 15 The Brain Trauma Foundation Guidelines for the surgical management of TBI, both surgical EDHs and subdural hematomas (SDHs), state that epidural hematomas should be operated on as soon as possible. ${ }^{1,2}$ This recommendation is based on a number of studies that found a positive association between better outcome and shorter delay to surgery.

For acute SDH, Seelig et al. ${ }^{19}$ found that the earlier the evacuation of the SDH after the trauma, the lower the mor-

ABBREVIATIONS ED = emergency department; EDH = epidural hematoma; GCS = Glasgow Coma Scale; GOS = Glasgow Outcome Scale; ISS = Injury Severity Score; $\mathrm{OR}=$ operating room; $\mathrm{SDH}=$ subdural hematoma; $\mathrm{TBI}$ = traumatic brain injury.

SUBMITTED January 24, 2015. ACCEPTED June 30, 2015.

INCLUDE WHEN CITING Published online January 1, 2016; DOI: 10.3171/2015.6.JNS15175. 
tality rate and the higher the chances of good recovery. A good recovery was achieved in $73 \%$ of the patients who underwent surgery within 2 hours of the trauma, but was seen in only $5 \%$ of those who underwent surgery more than 6 hours after the trauma, and the overall mortality rate was $30 \%$ if surgery was performed within 4 hours of the trauma, compared with $90 \%$ when done after 4 hours. Similarly, Haselsberger et al. ${ }^{6}$ showed that a delay of less than 2 hours between the onset of coma and surgery was associated with a mortality rate of $47 \%$, with good outcome in $32 \%$, compared with an $80 \%$ mortality rate and $4 \%$ with good outcome when the delay exceeded 2 hours.

For EDH, a study established a link between the onset of loss of consciousness and outcome, with only a 3\% mortality rate when the patients were conscious at the time of start of surgery. ${ }^{7}$ In patients who were initially conscious but became unconscious prior to surgery, however, the mortality rate was $28.6 \%$, similar to that for patients who had been unconscious since the onset of trauma. ${ }^{7}$ Likewise, Haselsberger et al. ${ }^{6}$ found that a delay of less than 2 hours between the onset of coma and surgery was associated with a $17 \%$ mortality rate and good recovery in $67 \%$, compared with $65 \%$ and $13 \%$, respectively, when the delay was more than 2 hours. In a similar study, Cohen et al. ${ }^{4}$ found that a delay of less than 90 minutes between anisocoria onset and surgery was associated with a better prognosis.

Sakas et al. ${ }^{17}$ correlated the delay between the onset of bilaterally unreactive pupils and surgery for mass lesion (SDH, EDH, and intracerebral hematoma). If the delay was less than 3 hours, the survival rate was $70 \%$, compared with $37 \%$ after 3 hours, and $0 \%$ after 6 hours. Lee et al..$^{12}$ and Seelig et al. ${ }^{20}$ found that the best motor response prior to surgery was 1 of the 2 main predictors of outcome in EDH (the other factor being associated brain injuries), favoring an early surgical evacuation before a clinical deterioration.

Only 1 study ${ }^{19}$ mentions the surgical technique used: a rapid temporal decompression with partial aspiration of the clot, followed by standard technique. Most studies used as a benchmark the time of patient arrival in the operating room. Some used the skin incision. Therefore, it is difficult to compare various time points. To date, to our knowledge, no study has looked at the actual timing of brain decompression in patients suffering from traumatic intracranial mass lesions. The goals of this study were as follows: 1) to characterize the performance of a level 1 trauma center in terms of delays for emergency trauma craniotomies; 2) to review step-by-step where the delays occurred in patient care; and 3) to propose ways to improve performance.

\section{Methods}

\section{Patient Population}

The Montreal General Hospital, part of the McGill University Health Centre, is an adult tertiary (Level 1) trauma center serving an adult population estimated at 2.8 million in 2011.

\section{Selection Criteria}

The operating room database was used to identify all patients for whom an emergency craniotomy was performed between April 1,2008, and March 31,2013, that was coded
"Priority 1" (i.e., the surgery has to be performed within 1 hour). Then, the Montreal General Hospital Traumatic Brain Injury Database and the Trauma Registry Database were used to confirm which patients suffered from TBIs. The next step was to confirm the status of emergency (i.e., a real Priority 1) by reviewing all the operative reports and all preoperative images. We subcategorized the patients into 2 groups: 1) E0 emergencies, defined as patients showing a massive lesion on CT imaging and significant compression or distortion of the brainstem, and/or a history of 1 or 2 dilated and fixed pupils secondary to the mass lesion; and 2) E1 emergencies, defined as patients with surgical mass lesions requiring urgent decompression but without anisocoria or significant brainstem compression. Investigators were blinded to the timing data when designating patients to either E0 or E1 categories. Patients who had nontraumatic pathologies or had only semiurgent surgical lesions, patients who needed surgery for late deterioration, and patients with missing data were excluded.

\section{Timing Calculation}

The following times were collected: arrival at the emergency department (ED time), CT imaging (CT time), arrival in the operating room (OR time), and the moment of skin incision (knife time). The ED time was determined by the arrival time recorded on the triage note. Arrival for CT imaging was defined as the time on the first image acquired (scout view). The OR time was defined as the time recorded in the OR database for arrival of the patient in the OR. The knife time was defined as the time recorded in the OR database for skin incision. We then calculated the delay between ED and CT times, between CT and OR times, between ED and OR times, between OR and knife times, and between ED and knife times. The duration of surgery and the total time in OR were also recorded.

Records of patients in E1 and E0 groups who had an interval between ED time and CT time of more than 2 hours (120 minutes) were reviewed to determine if there was a specific explanation for the prolonged delay. Data also were reviewed for patients in the E0 group who had a prolonged delay (CT-to-OR time longer than 45 minutes and OR-to-knife time longer than 60 minutes) once the diagnosis of a life-threatening mass lesion was made by CT imaging and clinical deterioration with 1 or 2 dilating pupils occurred.

\section{Surgical Technique}

The surgical reports were reviewed to classify the technique among the following: 1) standard 3- to 5-hole craniotomy/craniectomy, 2) rapid decompression with a large bur hole followed by a standard craniotomy/craniectomy, 3) posterior fossa craniectomy, and 4) penetrating head injury surgery (with spontaneous brain decompression from the penetrating injury site).

\section{Patient Characteristics}

The demographic data (i.e., age, sex) and injury characteristics (i.e., Glasgow Coma Scale [GCS] score, Injury Severity Score [ISS], mechanism of trauma, pupil dilation) were collected from the Montreal General Hospital TBI 
Database and the Trauma Registry Database. CT images were reviewed to define the main lesion requiring surgical intervention (i.e., $\mathrm{SDH}, \mathrm{EDH}$, intracerebral hematoma, intracerebral contusions, edema, penetrating head injury).

\section{Time of Day}

The patient's arrival time at the ED was classified as during regular business hours (Monday to Friday between 0800 and 1700 hours) or during evenings, nights, or weekends.

\section{Outcome Measures}

The primary outcome measure was the delay for each step of patient care. Secondary outcome measure was the Glasgow Outcome Scale (GOS) score ${ }^{8,11}$ at discharge from the acute care hospital and whether the discharge destination was home or another medical facility. The GOS score was always assigned according to a consensus within the multidisciplinary TBI team at discharge from the acute care hospital.

\section{Statistics}

Data were extracted from the OR, trauma, and TBI registries and merged, using SAS software (SAS Institute), on the basis of the record number and the surgery date. Additional data were recorded on a spreadsheet and incorporated. SAS JMP version 11 was used for analysis. Data distributions were described with nonparametric statistics. Continuous $x$ values (e.g., times) were compared with ordinal/nominal $y$ values with logistic fit. Nominal outcomes and continuous data were compared across categories by using the chisquare method and the median tests, respectively.

\section{Results}

\section{Emergency Craniotomies}

From April 1, 2008, to March 31, 2013, 408 craniotomies or craniectomies were performed. Of those, 246 were excluded, mainly because of delayed clinical or radiological deterioration (e.g., increased hematoma on follow-up CT scan, uncontrollable intracranial hypertension after a few days, postoperative recurrence of bleeding) (Fig. 1). The remaining 162 cases defined the study population. Of these, 104 (64\%) were classified as Priority 1 (E1) and 58 $(36 \%)$ as Priority 0 (E0) or extremely urgent. Most patients (73\%) arrived during evenings, nights, or weekends; $27 \%$ arrived during the regular business hours.

\section{Demographic and Injury Data}

Patients' characteristics are shown in Table 1.

\section{Timing}

The time intervals for all patients, as well as when they are divided into E0 and E1 groups, are given in Table 2. For patients who had a CT scan done in the local hospitals and who were then transferred to the Montreal General Hospital, the median ED-to-OR time was 75 minutes (interquartile range [IQR] 41-138 minutes). For patients brought directly to the Montreal General Hospital, the median ED-to-CT time was 54 minutes (IQR 42-74 minutes), and median CT-to-OR time was 57 minutes (IQR 31-87 minutes). The median ED-to-CT time interval was not significantly different between E0 and E1 groups. However, the ED-to-CT time was significantly longer on Sundays (median 89 minutes; $\mathrm{n}=21$ ) versus the other days of the week (range 48-60 minutes; $p=0.03$ ).

Seven patients (7\%) in the E1 group had an interval between ED arrival and arrival for CT imaging of longer than 120 minutes. Five of these patients were initially neurologically intact but subsequently deteriorated. One was an elderly patient with significant comorbidities whose family held a decision-making discussion, causing a longer interval. One patient had neurological deficits upon presentation, yet there was an interval of 134 minutes before a CT scan was done. Three patients $(5 \%)$ in the E0 group also had an interval longer than 120 minutes be-

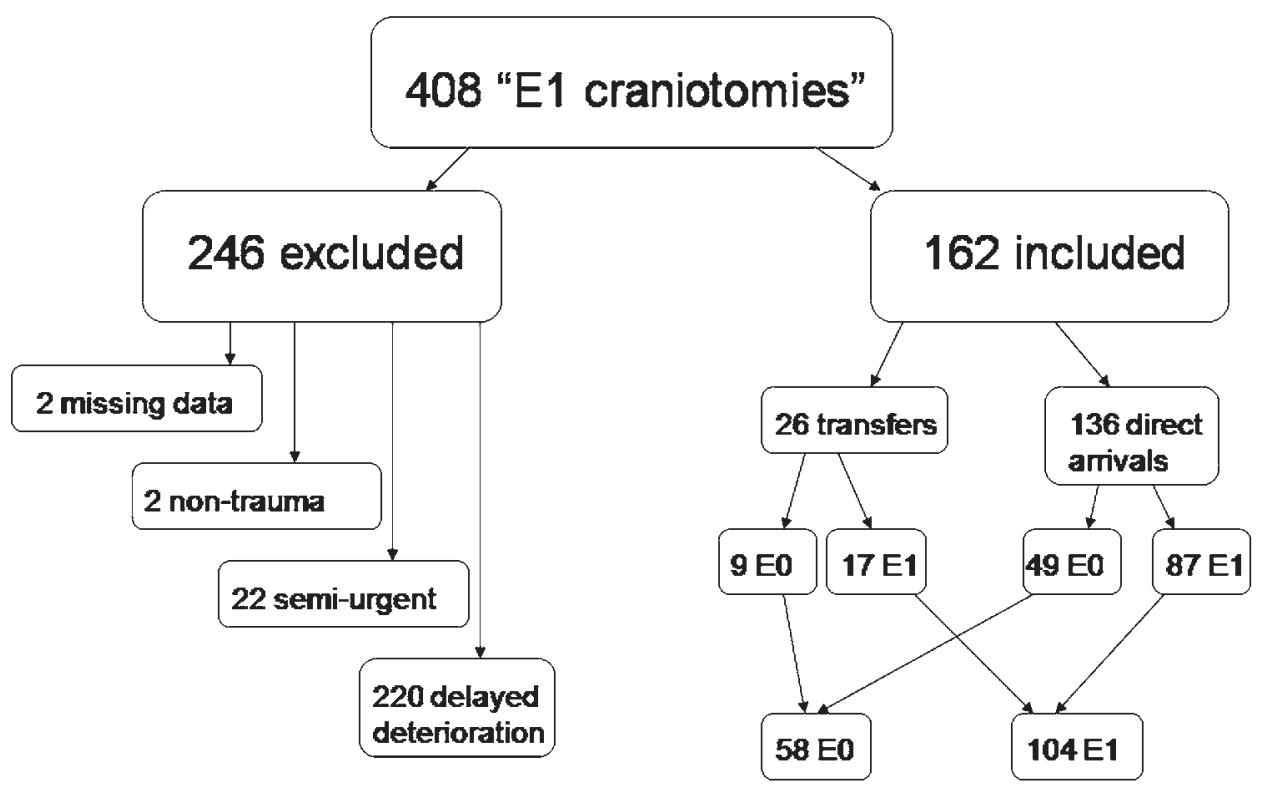

FIG. 1. Patient selection and exclusion process. 
TABLE 1. Patient characteristics

\begin{tabular}{lccc}
\hline \multicolumn{1}{c}{ Parameter } & Priority E0 $(\mathrm{n}=58)$ & Priority E1 $(\mathrm{n}=104)$ & Combined $(\mathrm{n}=162)$ \\
\hline Mean age, yrs (range) & $50(31-76)$ & $54(35-78)$ & $54(33-77)$ \\
\hline M/F, \% & $66: 34$ & $73: 27$ & $70: 30$ \\
\hline Mean ISS score (range) & $26(25-34)$ & $26(25-29)$ & $26(26-31)$ \\
\hline Mean RTS* (range) & $5.97(5.03-6.90)$ & $7.84(5.97-7.84)$ & $6.90(5.97-7.84)$ \\
\hline Mean RTS survival probability (range) & $0.77(0.48-0.87)$ & $0.88(0.73-0.86)$ & $0.87(0.63-0.95)$ \\
\hline Mean initial GCS score† (range) & $6(4-9)$ & $12(7-15)$ & $9(6-14)$ \\
\hline Hospital mortality rate, $\%$ & 53 & 29 & 38 \\
\hline Good outcome (GOS Score 5-6), \% & 27 & 51 & 44 \\
\hline RTS $=$ Revised Trauma Score. & & & \\
$*$ & & & \\
$\dagger<0.05$ between groups. & & &
\end{tabular}

tween their ED and CT times. Two of the 3 underwent anticoagulation treatment and needed their coagulation parameters to be corrected and 1 was an elderly patient with significant comorbidities.

There was a wide range in CT-to-OR times, with $10 \%$ of the patients being in the OR within 18 minutes of the scout scan and $10 \%$ requiring more than 120 minutes. The E0 group had a significantly shorter CT-to-OR time than the E1 group $(p=0.002)$. Of the 49 patients in the E0 group who had a CT scan performed at our institution, $21(43 \%)$ had a CT-to-OR time that exceeded 45 minutes. Of those 21 patients, 2 received anticoagulation treatment and needed their coagulation parameters corrected prior to surgery, and 1 was an elderly patient who had significant comorbidities and whose family held a discussion prior to making the decision to take the patient for surgery. Another of the 21 patients had a chronic component to his subdural hematoma, and 1 was hemodynamically unstable and required resuscitation prior to being taken to OR. No specific reason for a delayed transport to the OR could be found in the remaining 16 patients.

The time interval between the arrival in the OR and skin incision (OR-to-knife time) was significantly shorter for the E0 group than the E1 group ( $\mathrm{p}<0.0001)$. One patient in the E0 group had a interval between OR and incision that exceeded 60 minutes. That particular patient had a very large epidural hematoma, and yet it took 75 minutes from the time of arrival in the OR to the time of skin incision. In the E1 group, 2 patients had an OR-to-knife time

\section{TABLE 2. Time intervals*}

\begin{tabular}{|c|c|c|c|}
\hline Parameter & $\begin{array}{l}\text { Priority E0 } \\
(n=58)\end{array}$ & $\begin{array}{c}\text { Priority E1 } \\
(n=104)\end{array}$ & $\begin{array}{l}\text { Combined } \\
(n=162)\end{array}$ \\
\hline ED to CT imagingt‡ & $50(37-64)$ & $60(48-77)$ & $54(42-74)$ \\
\hline CT to OR† & $39(26-70)$ & 70 (39-92) & $57(31-87)$ \\
\hline ED to OR§ & $85(62-113)$ & $127(99-165)$ & $110(77-161)$ \\
\hline OR to knife§ & $27(20-35)$ & $39(30-45)$ & $35(25-45)$ \\
\hline ED to knife§ & $115(85-148)$ & $169(135-204)$ & $150(110-199)$ \\
\hline
\end{tabular}

\footnotetext{
* All data given as median minutes (IQR).

$\dagger$ Excluding patients having a CT scan in a local hospital and then transferred to the Montreal General Hospital.

$\ddagger p<0.05$ between groups.

$\S p<0.001$ between groups.
}

longer than 60 minutes. One had a time of 85 minutes but was in third-degree atrioventricular block and had an urgent transvenous pacemaker inserted prior to the craniotomy start. The other patient had an OR-to-knife time of 80 minutes, but no particular reason could be found for this prolonged installation time.

The time interval between ED arrival and OR arrival was also significantly shorter for the E0 group than the E1 group ( $\mathrm{p}<0.0001$ ), as was the interval from ED arrival to skin incision $(\mathrm{p}<0.001)$.

\section{Surgical Technique}

A standard craniotomy was used in $77 \%$ of the cases, while $17 \%$ had immediate bur hole decompression followed by craniotomy. The remaining patients had either posterior fossa craniectomies or penetrating brain injury surgeries. The proportion of patients with immediate bur hole decompression followed by completion of craniotomy climbed to $41 \%$ in the E0 group. Surgery lasted 120 minutes from skin incision to skin closure, on average (IQR 100-144 minutes). The median OR occupancy time was 175 minutes (IQR 152-200).

\section{Correlation Between Clinical Factors and Timing}

The interval from arrival in the ED to skin incision was 5.4 minutes faster for each point drop in GCS score $(\mathrm{p}<$ $0.0004)$. There was, however, no correlation between age and ED-to-knife times. There was no association between the presence of a significant chest injury (Abbreviated Injury Scale score of 3 or higher) and median ED-to-knife time (130 minutes [IQR 98-178 minutes]) with chest injury versus a median of 150 minutes (IQR 110-200 minutes) without chest injury. The same was observed for abdominal injuries: a median of 130 minutes (IQR 115-220 minutes) with an associated abdominal injury versus a median of 150 minutes (IQR 110-199 minutes) without injury. There was no correlation between the number of associated injuries, ISS, hemodynamic stability (i.e., blood pressure on arrival), and the speed of dealing with these emergency patients.

\section{Discussion}

To our knowledge, our study is the first to look at the time spent for each step in the care of acute surgical TBIs. 
Patients with obvious signs of clinical or radiological herniation (E0 group) did not undergo CT scanning faster, but the delay to get to the OR once the CT imaging was done and to make an incision was much shorter than for patients without obvious herniation (E1 group). The surgical technique used for herniated patients still most commonly followed a standard craniotomy opening style, yet immediate bur hole decompression was used more frequently in this group.

In the landmark paper by Seelig et al. ${ }^{19}$ on reduced mortality rates for SDH treated within 4 hours, a rapid temporal decompression with partial aspiration of the clot, followed by the standard craniotomy opening technique, was used for all patients. Rapid decompression can, indeed, save precious time for a herniating patient and, in our opinion, should have been used more frequently in our patient cohort. However, our delay in getting patients to the OR compares favorably to what is reported in the literature, with a median of 150 minutes from ED arrival to skin incision, and 110 minutes from ED arrival to arrival in the OR. The reported median delay by Wright et al. ${ }^{25}$ from ED to "neurosurgery" was 177 minutes.

For the general trauma population, delay in treatment has been identified as one of the major causes of preventable death. ${ }^{23}$ Furthermore, delay in transferring severely injured patients directly to a trauma facility has been shown to increase their mortality and morbidity. ${ }^{18}$ Another study, looking at all critically ill ED patients awaiting transfer to an intensive care unit, found that a delay of 6 or more hours for transfer was associated with a higher mortality rate, and a longer length of stay in the intensive care unit and in the hospital. ${ }^{3}$

There are few data specifically addressing TBI cases once in a hospital with neurosurgical capacity; however, a few studies have looked at the impact of delayed transfer of TBI patients to a neurosurgical care facility. Stone et al. ${ }^{22}$ found that for acute SDH, the mortality rate of the patients who had transited through another hospital was $76 \%$, compared with $50 \%$ of the directly admitted patients. On a few occasions, immediate bur hole decompression by a trauma surgeon for rapidly deteriorating patients, prior to transfer to a neurosurgical facility, had been shown to save patients' lives with a good functional outcome, ${ }^{16}$ but these few examples further highlight the fact that the sooner the decompression can be accomplished, the better the chances of survival and favorable outcome.

Causes of in-hospital delays for TBI patients have seldom been described in detail, not even for trauma patients at large. In comparison, delays between ED arrival and thrombolysis for stroke patients have received more attention. ${ }^{14,21}$ Patients arriving by emergency medical service instead of by their own means had shorter time to CT imaging ( 1 hour vs 1.3 hours; mean delay 1.1 hours, ${ }^{14} 134$ vs 215 minutes $^{21}$ ), and hospital arrival to physician ${ }^{11}$ and neurologist evaluation. ${ }^{21}$ In a systematic review ${ }^{10}$ that looked at ways to improve the time of thrombolysis delivery, training programs for emergency staff and reorganization of hospital systems, such as a "code stroke," proved efficient ways to reduce delays in treatment. In another study, one of the identified means to improve efficiency was the prehospital notification of a team. ${ }^{24}$ Prehospital notification is associated with impressive reductions in ED-to-CT time from 63 to 7 minutes. ${ }^{13}$

Although there is no Level 1 evidence in the literature that a faster evacuation of a mass lesion in the setting of TBI will result in better outcome, the current recommendation from the Brain Trauma Foundation is to evacuate a mass lesion in a comatose or deteriorating TBI patient as quickly as possible. ${ }^{1,2}$ This is based on many reports showing a better survival with timely decompression. ${ }^{4-6,7,12,17-19,22,25}$ To achieve the shortest delay possible, many players will have a role. An organized trauma system with quick transportation to a neurosurgical facility ${ }^{18,22,25}$ is a first step. Prenotification of the trauma team, including the neurosurgeon, similar to what occurs for myocardial infarctions or stroke cases, may help decrease delays once the patient arrives in the ED. Indeed, the rapid involvement of the attending neurosurgeon, in our opinion, can help decrease delays by more quickly making the decision to perform surgery. Bypassing the usual chain of command (i.e., junior resident to senior to neurosurgeon) can avoid, at times, unnecessary multiple neurological examinations and withholding of sedation (which could also increase intracranial pressure and brain damage). Working in concert with a trauma team leader to postpone less-urgent investigations (e.g., CT imaging of the chest and abdomen in a patient hemodynamically stable) and rather quickly transferring a patient with a growing intracranial mass lesion to the OR instead can also help reduce delays. Once in the OR, a tandem approach with the anesthesia team should prevail: While the neurosurgery team is preparing the patient's head, the anesthesia team is inserting intravenous catheters and establishing monitoring. Finally, an initial bur hole opening to quickly decompress the herniating brain, followed by the standard craniotomy, will also contribute to shorter delays in decompressing the intracranial compartment.

The present data suggest that fast ED-to-CT, CT-to-OR, and OR-to-knife times can be achieved. This is shown by the E0 group having a much shorter CT-to-OR time than the E1 group, and the correlation between decreasing GCS score and decreasing ED to OR times. Obviously, there are delays that are unavoidable (e.g., obtaining a CT scan when a patient initially presents neurologically intact, achieving hemodynamic stability, initiating correction of abnormal coagulation parameters, difficult decision dilemmas and family discussions, transport time). However, there are often interfering delays that cost precious minutes. In many instances, the delay to get the patient from CT imaging to the OR is very long and is possibly explained by the fact that the patient has further investigation (e.g., C-spine CT, chest, and abdomen scanning) or is simply brought back to ED and the neurosurgery service is only contacted afterward, instead of going directly to the OR from CT imaging.

The major weakness of this study is that the data were collected retrospectively and the causes of delays were not always captured (e.g., hemodynamic instability, need for other urgent investigation and treatment, difficult airway management). Also there could be some errors in the databases that were used and nonsynchronous time capture between the different systems. A much more accurate rootcause analysis would be obtained by prospectively collect- 
ing the times for each step. Nonetheless, we believe that this first report on the subject will provide some insight into the delays moving from 1 step to the next and help provide a plan for better performance and patient care to achieve a better survival for those patients who are potentially salvageable.

\section{Conclusions}

A timely evacuation of an intracranial mass lesion is a quality indicator for trauma care. Yet, a long delay for surgery still exists for patients requiring urgent mass lesion evacuation. Many factors contribute to these delays, including imaging, team notification/communication, decision making, transfer to the OR, preparation in the OR, and surgical technique. Strategies can be implemented to reduce delays and improve the delivery of care and patient outcome.

\section{References}

1. Bullock MR, Chesnut R, Ghajar J, Gordon D, Hartl R, Newell DW, et al: Surgical management of acute epidural hematomas. Neurosurgery 58 (3 Suppl):S7-S15, Si-iv, 2006

2. Bullock MR, Chesnut R, Ghajar J, Gordon D, Hartl R, Newell DW, et al: Surgical management of acute subdural hematomas. Neurosurgery 58 (3 Suppl):S16-S24, Si-iv, 2006

3. Chalfin DB, Trzeciak S, Likourezos A, Baumann BM, Dellinger RP: Impact of delayed transfer of critically ill patients from the emergency department to the intensive care unit. Crit Care Med 35:1477-1483, 2007

4. Cohen JE, Montero A, Israel ZH: Prognosis and clinical relevance of anisocoria-craniotomy latency for epidural hematoma in comatose patients. J Trauma 41:120-122, 1996

5. Gomez CR: Editorial: Time is brain! J Stroke Cerebrovasc Dis 3:1-2, 1993

6. Haselsberger K, Pucher R, Auer LM: Prognosis after acute subdural or epidural haemorrhage. Acta Neurochir (Wien) 90:111-116, 1988

7. Jamieson KG, Yelland JDN: Extradural hematoma. Report of 167 cases. J Neurosurg 29:13-23, 1968

8. Jennett B, Bond M: Assessment of outcome after severe brain damage. Lancet 1:480-484, 1975

9. Jennett B, Snoek J, Bond MR, Brooks N: Disability after severe head injury: observations on the use of the Glasgow Outcome Scale. J Neurol Neurosurg Psychiatry 44:285293, 1981

10. Kwan J, Hand P, Sandercock P: Improving the efficiency of delivery of thrombolysis for acute stroke: a systematic review. QJM 97:273-279, 2004

11. Lacy CR, Suh DC, Bueno M, Kostis JB: Delay in presentation and evaluation for acute stroke: Stroke Time Registry for Outcomes Knowledge and Epidemiology (S.T.R.O.K.E.). Stroke 32:63-69, 2001

12. Lee EJ, Hung YC, Wang LC, Chung KC, Chen HH: Factors influencing the functional outcome of patients with acute epidural hematomas: analysis of 200 patients undergoing surgery. J Trauma 45:946-952, 1998

13. Lindsberg PJ, Häppölä O, Kallela M, Valanne L, Kuisma M, Kaste M: Door to thrombolysis: ER reorganization and reduced delays to acute stroke treatment. Neurology 67:334336,2006

14. Morris DL, Rosamond W, Madden K, Schultz C, Hamilton S: Prehospital and emergency department delays after acute stroke: the Genentech Stroke Presentation Survey. Stroke 31:2585-2590, 2000

15. Pickard JD, Bailey S, Sanderson H, Rees M, Garfield JS:
Steps towards cost-benefit analysis of regional neurosurgical care. BMJ 301:629-635, 1990

16. Rinker CF, McMurry FG, Groeneweg VR, Bahnson FF, Banks KL, Gannon DM: Emergency craniotomy in a rural Level III trauma center. J Trauma 44:984-990, 1998

17. Sakas DE, Bullock MR, Teasdale GM: One-year outcome following craniotomy for traumatic hematoma in patients with fixed dilated pupils. J Neurosurg 82:961-965, 1995

18. Sampalis JS, Denis R, Fréchette P, Brown R, Fleiszer D, Mulder D: Direct transport to tertiary trauma centers versus transfer from lower level facilities: impact on mortality and morbidity among patients with major trauma. J Trauma 43:288-296, 1997

19. Seelig JM, Becker DP, Miller JD, Greenberg RP, Ward JD, Choi SC: Traumatic acute subdural hematoma: major mortality reduction in comatose patients treated within four hours. N Engl J Med 304:1511-1518, 1981

20. Seelig JM, Marshall LF, Toutant SM, Toole BM, Klauber MR, Bowers SA, et al: Traumatic acute epidural hematoma: unrecognized high lethality in comatose patients. Neurosurgery 15:617-620, 1984

21. Sekoranja L, Griesser AC, Wagner G, Njamnshi AK, Temperli P, Herrmann FR, et al: Factors influencing emergency delays in acute stroke management. Swiss Med Wkly 139:393-399, 2009

22. Stone JL, Lowe RJ, Jonasson O, Baker RJ, Barrett J, Oldershaw JB, et al: Acute subdural hematoma: direct admission to a trauma center yields improved results. J Trauma 26:445450, 1986

23. Teixeira PGR, Inaba K, Hadjizacharia P, Brown C, Salim A, Rhee $P$, et al: Preventable or potentially preventable mortality at a mature trauma center. J Trauma 63:1338-1347, 2007

24. Tveiten A, Mygland A, Ljøstad U, Thomassen L: Intravenous thrombolysis for ischaemic stroke: short delays and high community-based treatment rates after organisational changes in a previously inexperienced centre. Emerg Med J 26:324-326, 2009

25. Wright KD, Knowles CH, Coats TJ, Sutcliffe JC: 'Efficient' timely evacuation of intracranial haematoma-the effect of transport direct to a specialist centre. Injury 27:719-721, 1996

\section{Disclosures}

The authors report no conflict of interest concerning the materials or methods used in this study or the findings specified in this paper.

\section{Author Contributions}

Conception and design: Marcoux, Bracco. Acquisition of data: Marcoux, Bracco. Analysis and interpretation of data: all authors. Drafting the article: Marcoux. Critically revising the article: all authors. Reviewed submitted version of manuscript: all authors. Approved the final version of the manuscript on behalf of all authors: Marcoux. Statistical analysis: Bracco. Study supervision: Marcoux, Bracco.

\section{Supplemental Information \\ Previous Presentations}

A portion of this manuscript has been published in abstract form in the proceedings of the 49th Canadian Neurological Sciences Federation Congress, Banff, Canada, June 3-6, 2014.

\section{Correspondence}

Judith Marcoux, Department of Neurosurgery, Montreal General Hospital, McGill University Health Centre, 1650 Cedar Ave., Rm. L7-516, Montreal, Quebec H3G 1A4, Canada. email: judith. marcoux@mcgill.ca. 